\begin{tabular}{|c|l|}
\hline Title & Fano resonance in a multimode tapered fiber coupled with a microspherical cavity \\
\hline Author(s) & Chiba, A kito; Fujiwara, Hideki; Hotta, Jun-ichi; Takeuchi, Shigeki; Sasaki, Keiji \\
\hline Citation & $\begin{array}{l}\text { A pplied Physics Letters, 86, 261106 } \\
\text { https://doi.org/L0.1063/1951049 }\end{array}$ \\
\hline Issue Date & 2005-06-21 \\
\hline Doc URL & http://hdl.handle.net/2115/5551 \\
\hline Rights & Copyright $\odot 2005$ A merican Institute of Physics \\
\hline Type & article \\
\hline File Information & APL 86-26.pdf \\
\hline
\end{tabular}

Instructions for use 


\title{
Fano resonance in a multimode tapered fiber coupled with a microspherical cavity
}

\author{
Akito Chiba, ${ }^{a)}$ Hideki Fujiwara, Jun-ichi Hotta, Shigeki Takeuchi, and Keiji Sasaki \\ Research Institute for Electronic Science, Hokkaido University, N12W6, Sapporo 060-0812, Japan
}

(Received 11 April 2005; accepted 23 May 2005; published online 21 June 2005)

\begin{abstract}
Fano resonance in a tapered optical fiber in contact with a high- $Q$ microsphere is demonstrated. Multimode waves propagating in a $2.3 \mu \mathrm{m}$ diameter taper were coupled with a single whispering gallery mode of a $220 \mu \mathrm{m}$ sphere, and their coherent interaction resulted in Fano resonance. The asymmetric line shapes of the transmission spectra changed periodically with scanning of the coupling position along the taper. The observed $24 \mu \mathrm{m}$ period was due to modal dispersion in the tapered fiber. () 2005 American Institute of Physics. [DOI: 10.1063/1.1951049]
\end{abstract}

Fano resonance, originating in the interference between a discrete energy state and a continuum of states, is observed as a characteristically asymmetric line shape in transmission and reflection spectra. ${ }^{1,2}$ Anomalies occurring in diffractive grating and photonic crystal spectra have been explained by the Fano effect. ${ }^{3,4}$ Fano resonance leads to a drastic change in transmittance and reflectance over a narrow spectral range, this has application in fine frequency tuning, spectral filtering, light modulation, and highly sensitive sensors. ${ }^{5-7} \mathrm{Re}-$ cently, it has been shown that, in theory, Fano resonance can also be induced in a single-mode waveguide coupled with a microcavity. ${ }^{8}$ This has been demonstrated experimentally using a polymer microring resonator fabricated by nanoimprinting. ${ }^{9}$ In this waveguide/cavity coupling system, two partially reflecting elements are placed in the waveguide to control the phase difference between the resonant field and the transmitted wave, which is indispensable for the formation of the asymmetric line shape.

In this letter, we report that Fano resonance can be induced in a multimode tapered fiber waveguide coupled with a high- $Q$ microspherical cavity without the use of any additional elements, such as reflectors and delay optics. The tapered optical fiber, fabricated by heating and stretching a standard single-mode fiber, is a highly efficient coupling element for a microspherical resonator. ${ }^{10,11}$ By adjusting the taper diameter, the number of modes propagating in a tapered fiber can be restricted and the coupling modes of the tapered fiber and the microsphere can be selected. ${ }^{12,13}$

When the multimode waves in the taper are coupled to the microsphere with the resonant angular frequency $\omega_{c}$ and the free-spectral range $\nu_{c}$ (see Fig. 1), the cavity field at the position just before the coupling point is approximately given by

$$
E_{c}=\frac{i \alpha_{c} \nu_{c}}{\left(\omega-\omega_{c}\right)+i\left(1-\alpha_{c} t_{c}\right) \nu_{c}} \sum_{j} p_{j} E_{0},
$$

where $\alpha_{c}$ and $t_{c}$ are the round-trip factor and the transmittance in the coupling region of the microsphere, respectively. $E_{0}$ is the input field introduced into the fiber waveguide and $p_{j}(j=1,2, \ldots)$ are the coupling coefficients from the input field to the microsphere through the individual taper modes, that include the phase components depending on the propa-

\footnotetext{
${ }^{a)}$ Electronic mail: akichiba@es.hokudai.ac.jp
}

gation length. The output field from the fiber waveguide is expressed as

$$
E_{t}=\sum_{j}\left(t_{j} E_{0}+q_{j} E_{c}\right),
$$

where $t_{j}(j=1,2, \ldots)$ are the complex transmittances of the fields passing through the individual taper modes without coupling to the cavity and $q_{j}(j=1,2, \ldots)$ are the complex coupling coefficients from the cavity to the output field through the multimodes. From Eqs. (1) and (2), the intensity transmittance is given by

$$
\begin{aligned}
& T=\left|\frac{E_{t}}{E_{0}}\right|^{2}=\frac{|\bar{t}|^{2}\left(\omega-\omega_{c}\right)^{2}+C_{1}+C_{2}\left(\omega-\omega_{c}\right)}{\left(\omega-\omega_{c}\right)^{2}+\left(1-\alpha_{c} t_{c}\right)^{2} \nu_{c}^{2}}, \\
& C_{1}=\left|\left(1-\alpha_{c} t_{c}\right) \bar{t}+\alpha_{c} \bar{p} \bar{q}\right|^{2} \nu_{c}^{2}, C_{2}=2 \alpha_{c} \operatorname{Im}\left\lfloor\bar{t}(\bar{p} \bar{q})^{*}\right\rfloor \nu_{c},
\end{aligned}
$$

where $\bar{t}=\sum_{j} t_{j}, \bar{p}=\sum_{j} p_{j}, \bar{q}=\sum_{j} q_{j}$ and * denotes a complex conjugate. The first and second terms in the numerator of Eq. (3) represent a symmetric Lorentzian dip of the whispering gallery mode, while the last term exhibits an asymmetric line shape. In the case of a single-mode tapered fiber, the asymmetric component does not appear $\left(C_{2}=0\right)$, while the interaction among the multiple taper modes gives $C_{2} \neq 0$, which induces the Fano resonance.

When the coupling position is shifted by the distance $x$ along the fiber axis, $t_{j}$ is not changed but $p_{j}$ and $q_{j}$ are phase shifted as $\left.\bar{t}(\bar{p} \bar{q})^{*}\right|_{x}=\sum_{j, k,\{}\left\{\left.t_{j}\left(p_{k} q_{l}\right)^{*}\right|_{x=0} \exp \left(-i\left(\beta_{k}-\beta_{l}\right) x\right)\right]$, where

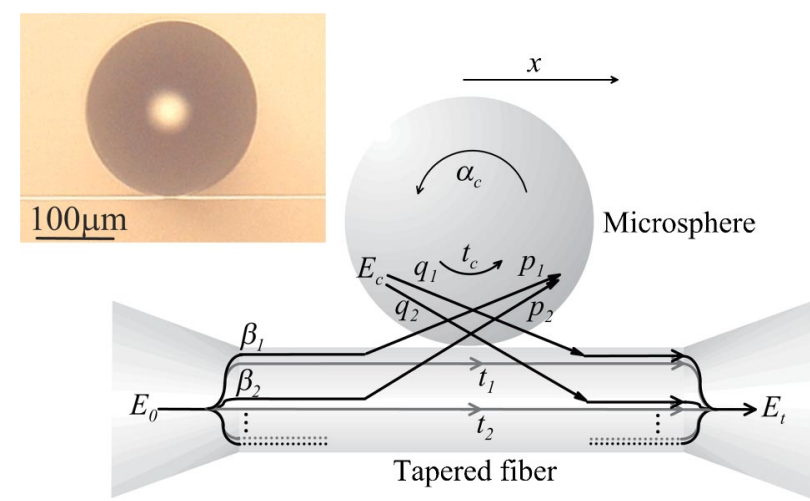

FIG. 1. (Color online) A sketch of a multimode tapered fiber waveguide coupled with a microspherical cavity. The microsphere was scanned along the tapered fiber in the direction indicated by arrow $x$. The inset shows a micrograph of the system. 


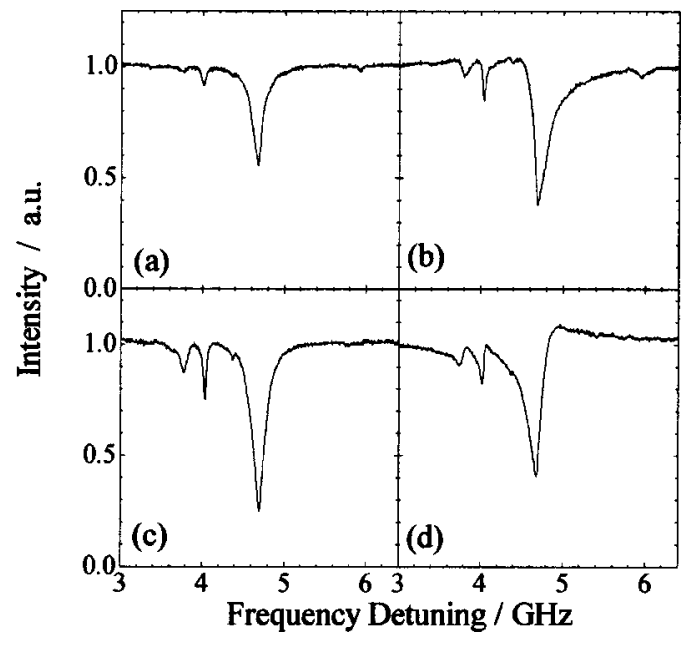

FIG. 2. Typical transmission spectra of a multimode tapered fiber in contact with a microspherical cavity measured at the coupling positions $x=$ (a) $0 \mu \mathrm{m}$, (b) $6 \mu \mathrm{m}$, (c) $12 \mu \mathrm{m}$, and (d) $18 \mu \mathrm{m}$. The spectra were normalized at offresonant frequency.

$\beta_{j}(j=1,2, \ldots)$ are the propagation constants of the taper modes. Supposing that two taper modes are coupled with the cavity, the asymmetric line shape is periodically changed with the shifting of the coupling position, with the period being given by $2 \pi\left|\beta_{1}-\beta_{2}\right|^{-1}$. It is noted that the coefficient $C_{1}$ containing the parameter $\bar{p} \bar{q}$ also changes with the displacement $x$.

A tapered fiber was prepared from a fused-silica singlemode optical fiber. After stripping the polymer coating, the fiber was heated using a ceramic heater and stretched into a fine thread with a waist diameter of $2.3 \mu \mathrm{m}$. This permitted the propagation of not only the fundamental propagation mode $\left(\mathrm{HE}_{11}\right)$, but also some higher-order propagation modes. ${ }^{12}$ Scanning electron micrographs indicated that there was no appreciable variation $(<0.1 \mu \mathrm{m})$ in the taper diameter over a $100 \mu \mathrm{m}$ length. A microsphere having a stem was also fabricated from a single-mode fiber as follows. The tip of a fiber from which the coating had been stripped was irradiated with a $\mathrm{CO}_{2}$ laser causing the fiber to melt and, due to surface tension, to form into a sphere. A sphere with a diameter of $220 \mu \mathrm{m}$ was used. The lowest radial-order modes of such a sphere satisfy the phase matching condition for coupling with the $\mathrm{HE}_{11}, \mathrm{HE}_{21}, \mathrm{TM}_{01}$ and $\mathrm{TE}_{01}$ modes of the $2.3 \mu \mathrm{m}$ tapered fiber. ${ }^{12,13} \mathrm{~A}$ micrograph of the spherical microcavity attached to the multimode tapered fiber is shown in the inset of Fig. 1.

A tunable external-cavity laser diode with a linewidth of $300 \mathrm{kHz}$ was coupled to the tapered fiber waveguide and the output light at the other end of the fiber was detected using a photodiode. The output intensity from the fiber when not coupled to the microsphere was $50 \mu \mathrm{W}$. The laser frequency was scanned over a range of $18 \mathrm{GHz}$ around a wavelength of $780 \mathrm{~nm}$ in order to observe the transmission spectra. The frequency was precisely calibrated by simultaneous measurement of rubidium vapor $\mathrm{D}_{2}$ lines as well as by means of a Fabry-Perot etalon (free spectral range $=2.5 \mathrm{GHz}$ ). The $5^{2} \mathrm{~S}_{1 / 2}(F=3) \rightarrow 5^{2} \mathrm{P}_{3 / 2}$ transition peak of ${ }^{85} \mathrm{Rb}$ was set to the origin of frequency detuning. In order to control the relative position of the sphere and the tapered fiber in three dimensions, the microsphere was mounted on a piezoelectric stage. The microsphere was kept in contact with the taper waist and Downloaded 21 Jun 2005 to 133.50 .96 .232 . Redistribution subject

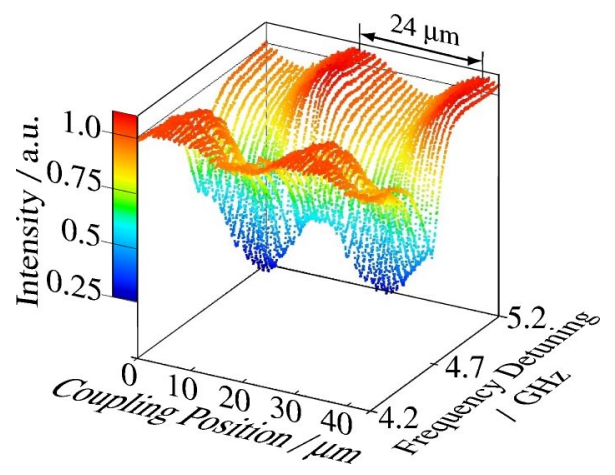

FIG. 3. (Color online) A three-dimensional plot of the position-dependent transmission spectra. For the spectral measurements, the coupling position was scanned at intervals of $1 \mu \mathrm{m}$ over a $45 \mu \mathrm{m}$ range.

the contact point was scanned along the tapered fiber. The microsphere/tapered-fiber-waveguide system was placed in a chamber which was filled with dry air (humidity $<1.0 \%$ ) to reduce water adsorption that may cause adhesion forces at the surfaces. The chamber was kept at a stable temperature, which is necessary to avoid any resonant frequency shift in the microspherical cavity. ${ }^{14}$

Figure 2 shows typical transmission spectra of the multimode tapered fiber/microsphere coupling system. These spectra were measured at different coupling positions, $x$ $=(\mathrm{a}) 0 \mu \mathrm{m}$, (b) $6 \mu \mathrm{m}$, (c) $12 \mu \mathrm{m}$, and (d) $18 \mu \mathrm{m}$. The spectral intensity was normalized using the signal at off-resonant frequency. The spectra exhibit an intense resonance at a detuning frequency of $4.7 \mathrm{GHz}$, as well as two small dips at 3.8 $\mathrm{GHz}$ and $4.0 \mathrm{GHz}$. The shapes of the resonant dips are dependent on the coupling position. The dip at $4.7 \mathrm{GHz}$ in Fig. 2(a) has a symmetric line shape with a linewidth of $160 \mathrm{MHz}$ corresponding to a $Q$ value of $2.4 \times 10^{6}$. Characteristically asymmetric Fano resonance line shapes were clearly observed in Figs. 2(b) and 2(d). One shoulder of the dip comprises a steep slope including an overshoot, while the other shoulder has a moderate slope followed by a long tail. The dip in Fig. 2(c) is symmetric and much deeper than that of Fig. 2(a). The width of the left-side shoulders, which are defined as the frequency width required for a change in transmission from $90 \%$ to $10 \%$ of the dip depth, are (a) $320 \mathrm{MHz}$, (b) $130 \mathrm{MHz}$, (c) $290 \mathrm{MHz}$ and (d) $550 \mathrm{MHz}$. The two small dips at 3.8 and $4.0 \mathrm{GHz}$, which possess $Q$ values of $4.1 \times 10^{6}$ and $1.0 \times 10^{7}$, respectively, exhibit the same change in shape as the dips described above, in spite of the difference in their widths and depths. It was confirmed that the spectral shapes did not change on increasing and decreasing the laser power. This indicates that, in the present experiment, thermal nonlinear effects caused by the introduction of the laser were negligibly small.

The coupling-position dependence of the resonant spectra is shown as a three-dimensional plot in Fig. 3. Transmission spectra were measured at every $1 \mu \mathrm{m}$ displacement within a scan range of $45 \mu \mathrm{m}$. The shapes of the spectra gradually change over the scan range. Clearly, the threedimensional plot shows a periodicity in the change in the spectra with respect to the displacement. The intensity at the dip and the side peaks varies sinusoidally with a period of $24 \mu \mathrm{m}$. The data were highly reproducible on backward scanning.

Theoretical analysis ${ }^{12}$ has shown that, at a $780 \mathrm{~nm}$ wavelength, the fundamental $\mathrm{HE}_{11}$ mode and the $\mathrm{HE}_{21}, \mathrm{TM}_{01}$, and
to AlP license or copyright, see http://apl.aip.org/apl/copyright.jsp 
$\mathrm{TE}_{01}$ modes of the $2.3 \mu \mathrm{m}$ tapered fiber possess propagation constants of $1.154 \times 10^{7} \mathrm{~m}^{-1}, 1.129 \times 10^{7} \mathrm{~m}^{-1}, 1.127 \times 10^{7}$ $\mathrm{m}^{-1}$, and $1.131 \times 10^{7} \mathrm{~m}^{-1}$, respectively. For the mode pairs $\mathrm{HE}_{11} / \mathrm{HE}_{21}, \mathrm{HE}_{11} / \mathrm{TM}_{01}$ and $\mathrm{HE}_{11} / \mathrm{TE}_{01}$, the calculated periods $2 \pi\left|\beta_{j}-\beta_{k}\right|^{-1}$ are $24.5 \mu \mathrm{m}, 23.1 \mu \mathrm{m}$, and $27.1 \mu \mathrm{m}$, respectively. Since a single whispering gallery resonance is either a TM or TE mode, coupling from both $\mathrm{TM}_{01}$ and $\mathrm{TE}_{01}$ taper modes into a single cavity mode of the microsphere cannot occur simultaneously. The experimental results show the period to be $24 \mu \mathrm{m}$, falling between 23.1 and $24.5 \mu \mathrm{m}$. This suggests that the dip observed in Fig. 3 can be ascribed to the TM microspherical resonance mode coupled with the $\mathrm{HE}_{11}, \mathrm{HE}_{21}$ and $\mathrm{TM}_{01}$ taper modes but not coupled with the $\mathrm{TE}_{01}$ wave. Furthermore, this suggests that the period is determined by the amplitude ratio between the $\mathrm{HE}_{21}$ and $\mathrm{TM}_{01}$ mode waves which are coupled to the cavity. The details of this analysis, which is based on the theory developed for multiple-input-output cavity systems, ${ }^{15}$ will be presented elsewhere.

In conclusion, Fano resonance in a multimode taper waveguide coupled with a microspherical cavity has been demonstrated. Dependent on the coupling position, the asymmetric line shape of the transmission spectrum was observed to change. This phenomenon is due to the modal dispersion in the tapered fiber and the multimode coupling between the taper and the microsphere. The steep transmission change originating from the high quality factor of a microsphere can be further enhanced by the Fano effect. In comparison to the single-mode-waveguide/cavity system using partially reflecting elements, ${ }^{8,9}$ the present system has the advantages of simplicity of device fabrication and controllability of the Fano resonance line shapes.
The authors would like to express sincere thanks to Professor H. F. Hofmann for useful discussions and to Professor K. Hakuta and Professor M. Kozuma for their advice regarding the experimental setup. This work was partially supported by the program "R\&D support scheme for funding selected IT proposals" of the Ministry of Public Management, Home Affairs, Posts, and Telecommunications and was also supported in part by Grants-in-aid for Scientific Research from the Japan Society for the Promotion of Science (JSPS) (13450024 and 15206007).

${ }^{1}$ U. Fano, J. Opt. Soc. Am. 31, 213 (1941).

${ }^{2}$ U. Fano, Phys. Rev. 124, 1866 (1961).

${ }^{3}$ D. Maystre, in Electromagnetic Surface Modes, edited by A. D. Boardman (Wiley, Chichester, UK, 1982).

${ }^{4}$ M. Kanskar, P. Paddon, V. Pacradouni, R. Morin, A. Busch, J. F. Young, S. R. Johnson, J. MacKenzie, and T. Tiedje, Appl. Phys. Lett. 70, 1438 (1997).

${ }^{5}$ G. L.-Yurista and A. A. Friesem, Appl. Phys. Lett. 77, 1596 (2000).

${ }^{6}$ A. Sharon, D. Rosenblatt, A. A. Friesem, H. G. Weber, H. Engel, and R. Steingrueber, Opt. Lett. 21, 1564 (1996).

${ }^{7}$ H.-T. Lee and A. W. Poon, Opt. Lett. 29, 5 (2004).

${ }^{8}$ S. Fan, Appl. Phys. Lett. 80, 908 (2002).

${ }^{9}$ C.-Y. Yen and L. J. Guo, Appl. Phys. Lett. 83, 1527 (2003).

${ }^{10}$ J. C. Knight, G. Cheung, F. Jacques, and T. A. Birks, Opt. Lett. 22, 1129 (1997).

${ }^{11}$ S. M. Spillane, T. J. Kippenberg, O. J. Painter, and K. J. Vahala, Phys. Rev. Lett. 91, 043902 (2003).

${ }^{12}$ A. W. Snyder and J. D. Love, Optical Waveguide Theory (Chapman and Hall, London, 1983).

${ }^{13}$ B. E. Little, J.-P. Laine and H. A. Haus, J. Lightwave Technol. 17, 704 (1999).

${ }^{14}$ A. Chiba, H. Fujiwara, J. Hotta, S. Takeuchi, and K. Sasaki, Jpn. J. Appl. Phys., Part 1 43, 6138 (2004).

${ }^{15}$ S. Fan, W. Suh, and J. D. Joannopoulos, J. Opt. Soc. Am. A 20, 569 (2003). 